(C) 1998 Technika

\title{
ALGORITHM OF GLOBAL UNIFORMIZATION OF ALGEBRAIC CORRESPONDENCE
}

\author{
O.B. DOLGOPOLOVA, E.I. ZVEROVICH \\ Belarusian State University, \\ F.Skaryna avenue 4, Minsk, 220050, Belarus \\ E-mail: OLGA@mmf.bsu.unibel.by
}

\begin{abstract}
The problem of global uniformization of algebraic correspondence is investigated. The weaker assumptions are used in the analysis.
\end{abstract}

\section{INTRODUCTION}

Let $f(z, w)$ be a polynomial of two complex variables over field $\mathbf{C}$ of all complex numbers. A correspondence between complex variables $z$ and $w$ is given by the following equation

$$
f(z, w)=0 .
$$

The problem of constructing a global uniformization of the correspondence (1) in an explicit form was investigated in [1] - [4]. In these papers, the problem was solved under assumption that the low for sewing sheets of the corresponding covering of the plane $\mathbf{C}_{z}$ is known and that the equation (1) is irreducible. It is known (see [5] - [7]) that the latter is equivalent to the connection of the Riemann surface $\Re$, which is determined by the equation (1). It was shown in [1] - [4] that, under this assumption, there exist a simply connected domain $D \subset \widehat{\mathbf{C}}$ with a piecewise smooth boundary $\partial D$ and there are functions

$$
\left\{\begin{array}{l}
z=\varphi(\xi) \\
w=\psi(\xi)
\end{array}\right.
$$

with the following properties: 
(a) one of the functions (2) is rational (and hence, another one is a branch of an algebraic function);

(b) $\forall \xi \in D \cup \partial D: f[\varphi(\xi), \psi(\xi)] \equiv 0$;

(c) the mapping $D \cup \partial D \rightarrow \Re$, which is determined by the equation (2), is surjective, and its restriction on the domain $D$ is injective.

Now we consider the problem of the global uniformization of the correspondence (1) without the assumptions mentioned above. In particular, we verify the equation (1) for the reducibility. We do not know any solution of this problem, since effective algorithms for calculation of the monodromy group of the equation (1) are not known.

\section{THE PROBLEM OF GLOBAL UNIFORMIZATION}

The equation (1) can be written in the form:

$$
f(z, w) \equiv a_{0}(z) w^{n}+a_{1}(z) w^{n-1}+\ldots+a_{n}(z)=0,
$$

where $a_{0}(z), a_{1}(z), \ldots, a_{n}(z)$ are polynomials. Assume without loss of generality that $a_{0}(z) \equiv 1$, since in the case of $a_{0}(z) \not \equiv 1$ we can introduce a new function $\zeta=a_{0}(z) w$, and the equation (3) will have the required form with respect to the variable $\zeta$. On the other hand, the use of variable $\zeta$ changes nothing, neither from the uniformization viewpoint nor from the reducibility viewpoint. We can find all branch points and all singular points of the equation (1) by solving the system of the algebraic equations

$$
\left\{\begin{aligned}
f(z, w) & =0 \\
\frac{\partial f(z, w)}{\partial w} & =0 .
\end{aligned}\right.
$$

Projecting these points on the plane $\mathbf{C}_{z}$ we assume that the number of distinct projections is equal to $m$. Let consider these projections in order of nondecreasing absolute values

$$
\left|z_{1}\right| \leq\left|z_{2}\right| \leq \ldots \leq\left|z_{m}\right| \leq+\infty
$$

Let $z_{m+1}=\infty$. We join these points sequentially by the smooth simple curves $L_{k}=\left[z_{k}, z_{k+1}\right], k=1, \ldots, m$, which do not have common inner points, and we denote $L=\bigcup_{k=1}^{m} L_{k}$. Let us take the direction from $z_{1}$ to $\infty$ as a positive direction on the curve $L$. The domain $D=\widehat{\mathbf{C}} \backslash L$ is simply connected. The compactification of the domain $D$ with respect to the Mazurkevich metric (see Fig. 1) is called the sheet: $D \cup \partial D=D \cup L^{+} \cup L^{-}$.

Choose a point $a \in D$ in such a way that the distance $r=\left|z_{1}-a\right|$ is less than the distances $\left|z_{2}-a\right|, \ldots,\left|z_{m}-a\right|$. Then in the disk $K=\{|z-a| \leq r\}$ 


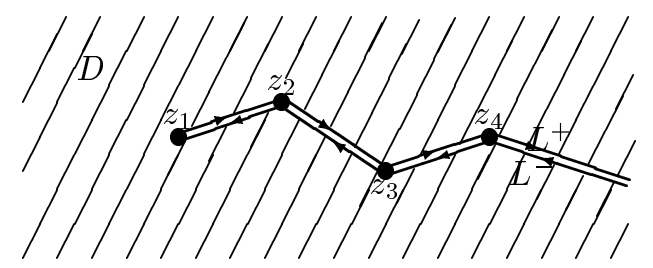

Figure 1.

there are no projections of singular points, nor projections of branch points of the equations (1); in particular, $\left.\frac{\partial f(z, w)}{\partial w}\right|_{z=a} \neq 0$. Therefore, there exist $n$ one-valued analytic functions

$$
w_{j}(z)=b_{j 0}+b_{j 1}(z-a)+b_{j 2}(z-a)^{2}+\ldots, j=1, \ldots, n,
$$

for which the equality $f\left[z ; w_{j}(z)\right] \equiv 0$ holds, where $|z-a| \leq r$. All the series (6) converge in the disk $|z-a| \leq r$ and their coefficients can be found sequentially by the "formal substitution".

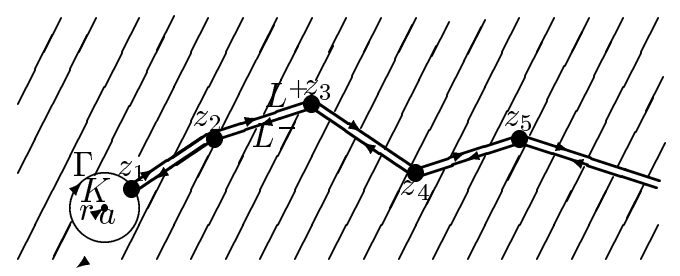

Figure 2.

Let us consider now the problem of the analytic continuation of the functions (6) from the disk $K$ onto the rest of the sheet $D \cup \partial D$. This problem can be solved by the reexpansion of the series (6) by powers of the differences $\left(z-a_{1}\right)$, where $a_{1} \neq a$. In such a way we can calculate the values of the functions in any point of the plane. However, this method gives only the fact of the existence of the monodromy group. If we want to calculate the monodromy group, then it is necessary to have a global formula for the analytic continuation of the functions (6) onto the whole sheet. It can be obtained by the method of the Carleman quenching function [8]. We apply this method here. Using the Newton diagram [6], we can find a natural number $k$ such that

$$
w_{j}(z)=O\left(z^{k-1}\right) \quad \text { where } z \rightarrow \infty
$$

for any $j=1, \ldots, n$. 
For this $k$, we can represent each functions $w_{j}(z)$ as a Cauchy-type integral over the boundary of the domain $G=D \backslash K$ (see Fig. 2):

$$
\begin{aligned}
& w_{j}(z)=\frac{1}{2 \pi i} \int_{\partial G} w_{j}(\tau) \frac{z^{k}}{\tau^{k}} \frac{d \tau}{\tau-z}=\frac{1}{2 \pi i}\left(\oint_{\Gamma}+\int_{L^{+}}-\int_{L^{-}}\right) w_{j}(\tau) \frac{z^{k}}{\tau^{k}} \frac{d \tau}{\tau-z}, \\
& j=1, \ldots n .
\end{aligned}
$$

However, in our case, the formula (7) gives nothing, because the values of the functions $w_{j}(z)$ are known only on the circle $\Gamma=\{|t-a|=r\}$ and are not known on the curves $L^{+}$and $L^{-}$. Carleman proposed "to quench" the influence of the integrals on the curves $L^{+}$and $L^{-}$. To do this, we find the function $\omega(z)$ which is harmonic on the domain $G$. We solve the following Dirichlet problem

$$
\omega(t)= \begin{cases}0, & t \in \Gamma \\ 1, & t \in L^{+} \cup L^{-} .\end{cases}
$$

Denote by $\tilde{\omega}(z)$ the function which is harmonic conjugate to $\omega(z)$. Then $\varphi(z)=\omega(z)+i \tilde{\omega}(z)$ is an analytic function in $G$, which is bounded for $z \rightarrow \infty$. Replacing $\omega_{j}(z)$ by $e^{-\nu \varphi(z)} w_{j}(z)$ in the formula (7), we obtain

$$
e^{-\nu \varphi(z)} w_{j}(z)=\frac{1}{2 \pi i}\left(\oint_{\Gamma}+\int_{L^{+}}-\int_{L^{-}}\right) e^{-\nu \varphi(\tau)} w_{j}(\tau) \frac{z^{k}}{\tau^{k}} \frac{d \tau}{\tau-z}, j=1, \ldots, n,
$$

from which we deduce

$$
w_{j}(z)=\frac{1}{2 \pi i} \lim _{\nu \rightarrow+\infty} \oint_{\Gamma} e^{\nu[\varphi(z)-\varphi(\tau)]} w_{j}(\tau) \frac{z^{k}}{\tau^{k}} \frac{d \tau}{\tau-z}, z \in G, j=1, \ldots, n,
$$

since the integrals over the curves $L^{ \pm}$tend to zero for $\nu \rightarrow+\infty$ uniformly with respect to $z \in T$, where $T \subset G$ is an arbitrary compact set. Thus the influence of unknown values of the functions $w_{j}(z)$ on the curves $L^{ \pm}$has been "quenched". We choose now $m$ ordinary points $a_{1} \in L_{1}^{0}, \ldots, a_{m} \in L_{m}^{0}$. By using the formula (8) and calculating limit values of right-hand part of (8) from the left and from the right in the points $a_{k} \in L_{k}^{0}$, we find two sets of pairwise distinct numbers:

$$
\left\{w_{j}^{+}\left(a_{1}\right), w_{j}^{+}\left(a_{2}\right), \ldots, w_{j}^{+}\left(a_{m}\right)\right\}
$$

and

$$
\left\{w_{j}^{-}\left(a_{1}\right), w_{j}^{-}\left(a_{2}\right), \ldots, w_{j}^{-}\left(a_{m}\right)\right\}, j=1, \ldots, n .
$$

Since the sets (9) and (10) must coincide, we can determine the permutation

$$
\sigma_{j}=\left(\begin{array}{cccc}
1 & 2 & \ldots & n \\
i_{j 1} & i_{j 2} & \ldots & i_{j n}
\end{array}\right), j=1, \ldots, n,
$$


where $i_{j k}$ is the index of the number from the sequence (10), which is equal to $w_{j}^{+}\left(a_{k}\right)$. The permutations (11) are the generators of the monodromy group of the equation (1). Finally, the equation (1) is reducible or is not reducible depending on whether the monodromy group acts transitively or not. In the latter case we can find the factorization of the polynomial $f(z, w)$ by expanding monodromy group into subgroups which act transitively.

Let us assume now that the monodromy group of the equation (1) is known and acts transitively. We consider the problem of the global uniformization, i.e., the problem of finding the functions (2). Our method is based on the next geometric idea. We find a closed curve $\Lambda$ on $n$-sheeted branched covering of the sphere $\widehat{\mathbf{C}}_{z}$ such that the open set $\Re \backslash \Lambda$ is simply connected. Furthermore, we find an inclusion of the set $\Re \backslash \Lambda$ into the closed Riemann surface $\mathcal{M}$, which is a finite-sheeted covering of the sphere $\widehat{\mathbf{C}}_{z}$ of genus zero. The desired uniformization may be expressed by the conformal homeomorphism of the surface $\mathcal{M}$ on the sphere $\widehat{\mathbf{C}}_{z}$. The homeomorphism can be constructed, for example, as an integral analogical to the Christoffel-Schwarz integral [1].

The construction of the surface $\mathcal{M}$ can be done either by attaching disks to the boundary of the compactification of the domain $\Re \backslash \Lambda$, or by gluing together the different parts of the boundary of this compactification. The latter procedure is more effective for calculations, because the number of sheets of the covering $\mathcal{M}$ is equal to the number of sheets of the covering $\Re$.

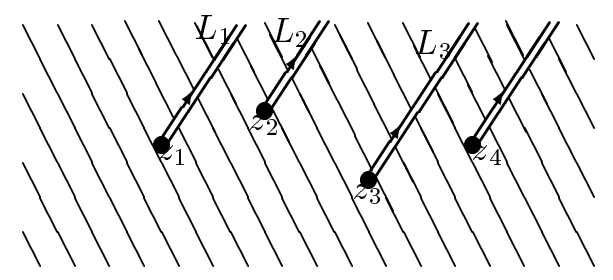

Figure 3.

To implement this method, we draw the cuts $L_{j}$ as the curves joining the points $z_{j}$ and $\infty$ (Fig. 3). For each curve $L_{j}$ there is a corresponding permutation from the monodromy group. Let there exist permutations containing transpositions, i.e.

$$
\sigma_{j}=\left(\begin{array}{lllll}
\ldots & k_{1} & \ldots & k_{2} & \ldots \\
\ldots & k_{2} & \ldots & k_{1} & \ldots
\end{array}\right)
$$

We make the following substitution:

$$
\sigma_{j} \mapsto \sigma_{j}^{*}, \text { where } \sigma_{j}^{*}=\left(\begin{array}{ccccc}
\ldots & k_{1} & \ldots & k_{2} & \ldots \\
\ldots & k_{1} & \ldots & k_{2} & \ldots
\end{array}\right),
$$

provided that in position of dots nothing was changed. After the above substitution a new group is obtained. If the new group does not act transitively, 
then we cancel the substitution. The branch index can decrease only as a result of the substitution. We sort out all transpositions and eliminate them if the substitutions $\sigma_{j} \mapsto \sigma_{j}{ }^{*}$ do not result in loss of transitivity. Then we examine permutations containing 3 -cycles:

$$
\sigma_{j}=\left(\begin{array}{lllllll}
\ldots & k_{1} & \ldots & k_{2} & \ldots & k_{3} & \ldots \\
\ldots & k_{2} & \ldots & k_{3} & \ldots & k_{1} & \ldots
\end{array}\right)
$$

For these permutations, we substitute

$$
\sigma_{j} \mapsto \sigma_{j}{ }^{*}, \text { where } \sigma_{j}{ }^{*}=\left(\begin{array}{ccccccc}
\ldots & k_{1} & \ldots & k_{2} & \ldots & k_{3} & \ldots \\
\ldots & k_{1} & \ldots & k_{3} & \ldots & k_{2} & \ldots
\end{array}\right)
$$

in such a way that exactly one of the numbers $k_{1}, k_{2}$ or $k_{3}$ does not change its position. We cancel the substitution if and only if it breaks transitivity. And again, the branch index can decrease only. Then we examine permutations containing 4-cycles, etc. Since the number of all permutations and the number of all cycles is finite, it follows that after a finite number of iterations any substitution $\sigma_{j} \mapsto \sigma_{j}{ }^{*}$ breaks transitivity. This means that the branch index decreases to $2(n-1)$, and the genus of the Riemann surface $\mathcal{M}$ formed by gluing the sheets is equal to zero.

\section{REFERENCES}

[1] Zverovich E.I., About the possibility of constructing global uniformization of algebraic correspondence in an explicit form, Vestnik BGU, 1, No.1, (1991), P. 36 - 39 (in Russian).

[2] Zverovich E.I., Set'ko E.A., Construction of global uniformization of algebraic correspondence in an explicit form, Deposit in VINITI 14.05.1991, No.1972-B91 (in Russian).

[3] Dolgopolova O.B., Zverovich E.I., Uniformization of algebraic correspondences. Proceedings of International Conference "Boundary value problems, special functions and fraction calculus". Minsk, BSU, (1996), P. 76 - 80 (in Russian).

[4] Dolgopolova O.B., Zverovich E.I., Construction of global uniformization of algebraic correspondences in an explicit form. Theses of International Conference "Boundary value problems, special functions and fraction calculus", Minsk, BSU, (1996), P. 35 (in Russian).

[5] Springer G., Introduction to Riemann surfaces, New York: Chelsea, (1981).

[6] Chebotarev N.G., Theory of algebraic functions, Moscow-Leningrad: Gostekhizdat, (1948) (in Russian).

[7] Nevanlinna R.H., Uniformisierung, Berlin: Springer, 1953.

[8] Aizenberg L.A., Carleman formulae in complex analysis, Novosibirsk: Nauka, (1990) (in Russian). 